\title{
Environmental Dependence of Thermal Reaction Norms: Host Plant Quality Can Reverse the Temperature-Size Rule
}

\author{
Sarah E. Diamond ${ }^{\star}$ and Joel G. Kingsolver \\ Department of Biology, University of North Carolina, Chapel Hill, North Carolina 27599 \\ Submitted June 3, 2009; Accepted August 12, 2009; Electronically published November 13, 2009 \\ Online enhancement:appendix.
}

\begin{abstract}
AвSTRACT: The temperature-size rule, a form of phenotypic plasticity in which decreased temperature increases final size, is one of the most widespread patterns in biology, particularly for ectotherms. Identifying the environmental conditions in which this pattern is reversed is key to understanding the generality of the rule. We use wild and domesticated populations of the tobacco hornworm Manduca sexta and the natural host plants of this species to explore the consequences of resource quality for the temperature-size rule. Manduca sexta reared on a high-quality host, tobacco (Nicotiana tabacum), followed the temperature-size rule, with larger final sizes at lower temperatures. In contrast, $M$. sexta reared on a low-quality host, devil's claw (Proboscidea louisianica), showed the reverse response. Wild and domesticated M. sexta exhibited qualitatively similar responses. Survival, growth and development rates, fecundity, and final size decreased with decreasing temperature in $M$. sexta reared on devil's claw. We propose that the reversal of the temperature-size rule results from the stressful combination of low temperatures and low dietary quality. Such reversals may impact seasonal and geographic patterns of host use in Manduca and other systems. Our results suggest that the temperature-size rule occurs for a restricted range of nonstressful environmental conditions, limiting the robustness of this widespread pattern of phenotypic plasticity.
\end{abstract}

Keywords: Manduca sexta, phenotypic plasticity, life-history trade-off, thermal reaction norm, diet quality, temperature-size rule.

\section{Introduction}

One of the most recognized forms of phenotypic plasticity is the relationship between body size and temperature (Atkinson 1994). In ectotherms, decreasing rearing temperature typically causes decreased growth and development rates but a larger final body size: a relationship that has been called the temperature-size rule. This generates a negative thermal reaction norm-the relationship between

\footnotetext{
* Corresponding author. Address for correspondence: Department of Biology, University of North Carolina, CB-3280, Coker Hall, Chapel Hill, NC 27599; e-mail: sdiamond@email.unc.edu.

Am. Nat. 2010. Vol. 175, pp. 1-10. (c) 2009 by The University of Chicago. 0003-0147/2010/17501-51321\$15.00. All rights reserved.

DOI: $10.1086 / 648602$
}

body size and rearing temperature-for final body size. Although substantial variation in thermal reaction norms has been documented among individuals, populations, and species, the temperature-size rule is one of the most taxonomically widespread patterns in biology; more than $80 \%$ of ectothermic species studied to date follow the temperature-size rule (Atkinson 1994). The phenotypic pattern described by the temperature-size rule is mirrored at the evolutionary level in another widespread empirical generalization known as Bergmann's rule: cooler climates tend to have populations or species with larger body sizes (Partridge and Coyne 1997; Blanckenhorn and Demont 2004).

Both adaptive and mechanistic explanations for the temperature-size rule have been proposed (Berrigan and Charnov 1994; van der Have and de Jong 1996; Davidowitz et al. 2004; Nijhout et al. 2006), but it remains unclear whether this pattern is produced by natural selection or shared physiological constraints. Often, proposed mechanistic constraints and life-history optimality models seeking to explain the temperature-size rule suffer from a lack of generality (Angilletta and Dunham 2003; Angilletta et al. 2004; Angilletta 2009). How do we reconcile the lack of a satisfactory general explanation for the temperaturesize rule with how commonly it occurs in nature?

Understanding exceptions to the temperature-size rule can be particularly informative (Angilletta and Dunham 2003; Walters and Hassall 2006; Kingsolver and Huey 2008). Exceptions have been reported in several taxa, but the only documented reversals of the temperature-size rule (i.e., positive thermal reaction norms for final body size) have been in insects, most commonly in Lepidoptera and Orthoptera (Atkinson 1994; Mousseau 1997). Reversal of the temperature-size rule in one geographic population of the butterfly Pieris rapae has likely evolved within the past 160 years, since the colonization of North America by this species (Kingsolver et al. 2007). Observed reversals of the temperature-size rule in grasshoppers can be produced by changing the relative positions of the minimum temper- 
ature coefficients for growth versus development rates (Walters and Hassall 2006). In this case, the reversal of the temperature-size rule may emerge as a result of selection for a greater temperature threshold for growth versus development or as a by-product of selection for greater thermal specialization in growth rate (Huey and Hertz 1984; Walters and Hassall 2006).

An important issue in exploring the temperature-size rule and its exceptions is how temperature may interact with other environmental factors that affect growth, development, and final size (Berrigan and Charnov 1994). For example, numerous studies show that reductions in the resource (diet) quality result in reduced growth and development rates, delayed development times, and smaller final body sizes (e.g., Stearns and Koella 1986) and that resource quality is a major determinant of resource use in nature (Awmack and Leather 2002). In contrast, reduced rearing temperature typically results in reduced growth and development rates and delayed development time but larger final body sizes, that is, the temperaturesize rule (Davidowitz et al. 2004). In some cases, changes in nutritional quality and secondary compounds can alter the slope of the thermal reaction norm for size (Stamp 1990, 1994; Yang and Stamp 1996; Kingsolver et al. 2006). If diet quality can alter thermal reaction norms, then this may impact food choice and resource use in different seasons or climatic regions. How, then, do temperature and diet quality interact? Is the temperature-size rule robust to environmental variation in diet quality?

We explore these questions using the tobacco hornworm Manduca sexta (Lepidoptera: Sphingidae), an important model system for the study of insect growth and development. When reared on an artificial diet, $M$. sexta follows the temperature-size rule across a range of thermal conditions $\left(\sim 20^{\circ}-35^{\circ} \mathrm{C}\right.$; Davidowitz et al. 2004; Kingsolver and Nagle 2007). In nature, M. sexta feeds on a range of host plant species that vary in quality, but the consequences of this variation in diet quality for the shape of thermal reaction norms have not been explored. Here we focus on two food resources: a high-quality host plant, tobacco ( $\mathrm{Ni}$ cotiana tabacum; family Solanaceae), and a low-quality host plant, devil's claw (Proboscidea louisianica; family Martyniaceae). Previously, we documented substantial fitness costs in the use of devil's claw by M. sexta: survival, growth rate, development rate, and fecundity are all reduced when $M$. sexta is reared on this host plant species compared with when M. sexta is reared on tobacco (S. E. Diamond, S. D. Hawkins, H. F. Nijhout, and J. G. Kingsolver, unpublished manuscript).

In this article, we compare responses of two populations of M. sexta: a wild field population and a domesticated laboratory population. The domesticated population was derived from this same field population over 40 years ago, representing more than 250 generations in the laboratory. These two populations have important differences in their thermal and dietary histories. The domesticated laboratory population has been maintained under a constant $25^{\circ} \mathrm{C}$ thermal regime and reared on an artificial diet since its introduction to the laboratory. In contrast, the wild field population experiences diurnally and seasonally fluctuating temperatures and feeds on a variety of natural host plants. As a consequence of laboratory domestication, the laboratory population exhibits greater thermal sensitivity, particularly at warmer temperatures $\left(35^{\circ} \mathrm{C}\right)$, relative to the wild field population (Kingsolver and Nagle 2007; Kingsolver et al. 2009).

In this study, we experimentally examine whether the temperature-size rule is robust to environmental variation in diet quality. To accomplish this, we draw intraspecific comparisons, replicated across two populations, in which we explore the consequences of the interaction between temperature and diet quality for final body size. We address three major questions: (1) How do temperature and diet quality interact to affect survival, development time, and fecundity? (2) Does host plant quality affect the temperature-size rule? (3) Do population responses differ in the way host plant quality affects survival, development time, fecundity, and the temperature-size rule? Here, fecundity provides a crucial link between body size and fitness: adaptive explanations for the temperature-size rule generally rely on a positive relationship between size and fecundity (Atkinson and Sibly 1997). Our results demonstrate that the combination of poor diet quality and low temperatures is stressful for M. sexta, contributing to prolonged development times and reductions in survival and fecundity. We show that the temperature-size rule can be reversed under these stressful conditions, reversing the slope of thermal reaction norms for final body size. This reversal may impact seasonal and geographic patterns of host use in Manduca and, perhaps, in other study systems.

\section{Material and Methods Study System}

The tobacco hornworm Manduca sexta L. (Sphingidae) is distributed across tropical and temperate regions of the Nearctic (Rothschild and Jordan 1903). Larval feeding is generally restricted to plants in the Solanaceae, and $M$. sexta is an important agricultural pest of tobacco (Nicotiana tabacum: Solanaceae) in the southeastern United States.

Recently M. sexta has been documented to also use devil's claw (Proboscidea spp.: Martyniaceae) as a host plant in the southwestern United States (Mechaber and Hildebrand 2000; Mira and Bernays 2002). Proboscidea 
species are native to the Southwest but have been introduced to other locations across the United States. One species of devil's claw, Proboscidea louisianica (Miller) Thellung, has been naturalized to the southeastern United States (Small 1903). Proboscidea louisianica is patchily distributed across North Carolina, compared with the predominant local solanaceous host plant, tobacco (Nicotiana tabacum L.), which is widely cultivated across the state (Radford et al. 1968). Despite the limited distribution of devil's claw in North Carolina, our observations indicate that field populations of $M$. sexta oviposit and feed successfully on this host plant when it is grown adjacent to tobacco plants (S. E. Diamond, S. D. Hawkins, H. F. Nijhout, and J. G. Kingsolver, unpublished manuscript).

Manduca sexta has reduced survival, growth rate, development rate, and fecundity when reared at intermediate temperatures $\left(25^{\circ} \mathrm{C}\right)$ on devil's claw compared with on tobacco (S. E. Diamond, S. D. Hawkins, H. F. Nijhout, and J. G. Kingsolver, unpublished manuscript). Here we consider devil's claw ( $P$. louisianica) to be a low-quality resource and a low-alkaloid variety of cultivated tobacco (N. tabacum, var. LA Burley 21) to be a high-quality resource. Tobacco seeds were obtained from the National Genetic Resources Program (USDA, Agricultural Research Service, Beltsville, MD).

We used two genetic lines (populations) of $M$. sexta in our experiments. The laboratory population came from a colony maintained under standard larval rearing conditions (artificial diet, constant temperature of $25^{\circ}-26^{\circ} \mathrm{C}$, 15L : 9D photocycle) by L. Gilbert and colleagues at University of North Carolina for over 25 years. This colony is ultimately derived from field collections of eggs in Clayton, North Carolina (North Carolina State University Research Station) during the 1960s. Field-population eggs used in the current experiments were offspring of adults that were collected as early-instar larvae from this same Clayton, North Carolina, field site and reared through one generation on an artificial diet in the laboratory to minimize parental effects.

\section{Experiments}

Eggs from each population (laboratory and field) were randomly assigned to tobacco and devil's claw leaves held under three different constant temperature regimes $\left(20^{\circ}\right.$, $25^{\circ}$, and $30^{\circ} \mathrm{C}$ ) in the laboratory. We employed a fully factorial design: there were 12 different treatment groups representing all combinations of population (field, laboratory), host plant species (devil's claw, tobacco), and temperature $\left(20^{\circ}, 25^{\circ}, 30^{\circ} \mathrm{C}\right)$. A previous study (at $25^{\circ} \mathrm{C}$ ) demonstrated greater larval survival on tobacco compared with on devil's claw (S. E. Diamond, S. D. Hawkins, H. F. Nijhout, and J. G. Kingsolver, unpublished manuscript).
Therefore, upon hatching, 20 larvae from each population were transferred to individual tobacco leaves in each of the three temperature treatments, and a larger number of larvae (30-50) from each population were transferred to individual devil's claw leaves in each of the three temperature treatments.

Larvae were maintained individually in large petri dishes (2.5-cm height $\times 14.5-\mathrm{cm}$ diameter) housed in environmental chambers (Percival 36-VL) under a 16L : 8D photocycle. Larvae remained in petri dishes until the fourth larval instar, when they were transferred to larger plastic enclosures $(31 \mathrm{~cm} \times 16 \mathrm{~cm} \times 13 \mathrm{~cm})$ with screened lids. Larvae were fed ad lib. on whole leaves cut from greenhouse-grown plants. Leaf water content was maintained by placing the leaves in water picks. Leaves were changed every 2-3 days for early-instar larvae and every day for fifth-instar larvae. Leaf material was harvested from greenhouse-grown plants of the same age (4 weeks old at the start of the experiment). These plants were fertilized weekly with Peter's Pro Solution (15-16-17). We grew an excess of plants to minimize the effect of inducing secondary plant metabolites in response to repeatedly cutting leaves from individual plants. No pesticides were applied to these plants.

Larvae were maintained in growth chambers until wandering, when they were placed individually in wooden pupation chambers. After wandering, M. sexta larvae from all treatment groups were held under a constant $25^{\circ} \mathrm{C}$ thermal regime until eclosion. Pupae were transferred to plastic cups lined with soil, and they remained there until eclosion. We measured survival, development time, and body mass at the second, third, fourth, and fifth (sixth in some cases) larval instars, and wandering, pupation, and eclosion. Larval masses were recorded immediately following the molt into a given instar (indicated by slipping of the head capsule). Wandering masses were recorded following complete purging of the gut contents. Analyses of growth and development were restricted to individuals that survived to eclosion.

Adults were frozen at $-80^{\circ} \mathrm{C}$ at $24 \mathrm{~h}$ after eclosion. For adult females, estimates of potential fecundity were obtained by dissecting the ovarioles into Ringer's solution. The number of follicles at stage 6 and all subsequent stages of development (see Yamauchi and Yoshitake 1984 for staging follicles) were counted with the aid of a dissecting microscope (Nikon SMZ-1B).

\section{Statistical Analyses}

Survival to pupation was treated as a binomial variable and modeled using analysis of deviance with population (laboratory or field), host plant species (tobacco or devil's claw), temperature $\left(20^{\circ}, 25^{\circ}, 30^{\circ} \mathrm{C}\right)$, and all two- and three- 
way interactions between these terms as fixed effects. Here (and in all subsequent analyses), temperature was treated as a continuous covariate; treating temperature as a factor did not substantially alter any of our results. Development time to pupation and body mass at pupation (for individuals surviving to eclosion) were modeled using ANCOVA with population, host plant species, temperature, sex, and all two- and three-way interactions between these terms (exclusive of those interactions with sex) as fixed effects. We focused on measurements of pupae because of the stability in body mass at this stage. However, because all $M$. sexta were held at $25^{\circ} \mathrm{C}$ after reaching the wandering stage regardless of larval rearing temperature, we also examined body mass and development time at the wandering and adult stages to confirm our results. We also evaluated the effects of temperature and plant quality on potential fecundity on the basis of measurements of the number of follicles in ovarioles. Potential fecundity was modeled using ANCOVA with population, host plant species, temperature, and all two- and three-way interactions between these terms as fixed effects. Because body size has important effects on fecundity in M. sexta and other insects (Davidowitz et al. 2004, 2005), pupal mass was included as a continuous covariate. All statistical analyses were performed using R (ver. 2.8.1) statistical software.

\section{Results}

\section{Survival}

Relative to when tobacco was the host plant, mean survival was significantly reduced in Manduca sexta raised on devil's claw, particularly at cooler temperatures (fig. 1). Analysis of deviance detected significant effects of host plant species (tobacco or devil's claw; $\chi^{2}=96, P<$ $.0001)$ and temperature $\left(\chi^{2}=32, P<.0001\right)$ and a marginally significant effect of population (laboratory or field; $\chi^{2}=3.8, P=.052$ ) on the probability of survival to pupation. No significant two- or three-way interactions were found between host plant species, temperature, and population (plant $\times$ population: $\chi^{2}=0.91, P=.34$; plant $\times$ temperature: $\chi^{2}=0.81, P=.39$; population $\times$ temperature: $\chi^{2}=0.18, P=.67$; plant $\times$ population $\times$ temperature: $\chi^{2}=0, P=1$ ). In general, the laboratory population had slightly lower overall survival compared with the field population, as indicated by the significant main effect of population (fig. 1). Results were qualitatively similar for the probability of survival to adult eclosion.

To further explore the overall significant effect of temperature on survival, we performed post hoc analyses: for individuals on tobacco, temperature $\left(\chi^{2}=0, P=1\right)$ was not significant (nor were population $\left[\chi^{2}=1.4, P=.24\right]$ or the interaction between population and temperature

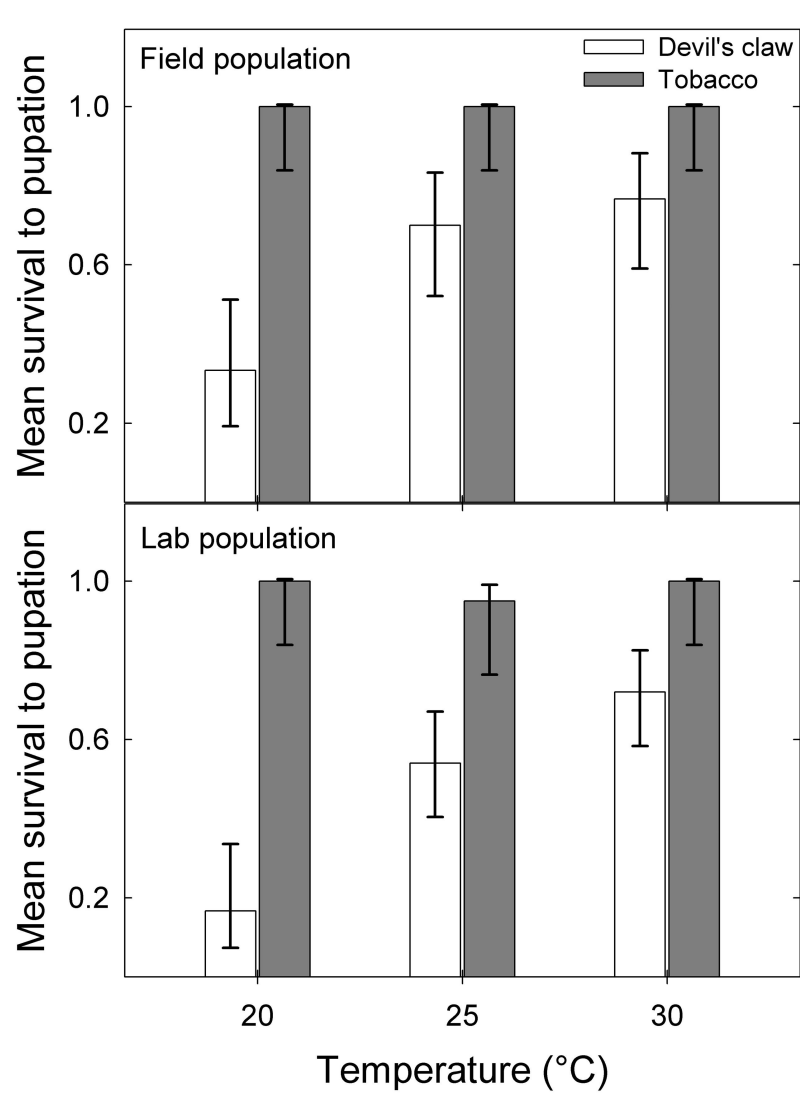

Figure 1: Mean survival to pupation $\pm 95 \%$ confidence interval for a field population (top) and a laboratory population (bottom) of Manduca sexta reared on devil's claw (white bars) and tobacco (gray bars) at $20^{\circ}$, $25^{\circ}$, and $30^{\circ} \mathrm{C}$.

$\left.\left[\chi^{2}=0, P=1\right]\right)$. In contrast, for individuals on devil's claw, temperature was significant $\left(\chi^{2}=33, P<.0001\right)$, but population $\left(\chi^{2}=3.3, P=.067\right)$ and the interaction of temperature and population $\left(\chi^{2}=0.28, P=.54\right)$ were not significant. Therefore, the significant effect of temperature in the full model was driven entirely by the results for devil's claw in which survival decreased with decreasing temperature, rather than by those results for tobacco in which survival was relatively constant across temperature treatments.

\section{Development and Growth}

Overall, there was greater variability in mean body mass and development time in the growth trajectories of $M$. sexta reared on devil's claw relative to tobacco (figs. 2, A1 in the online edition of the American Naturalist). Larval growth trajectories of individuals reared on tobacco were approximately exponential (linear on a natural log scale), with larvae generally growing at the same proportional 


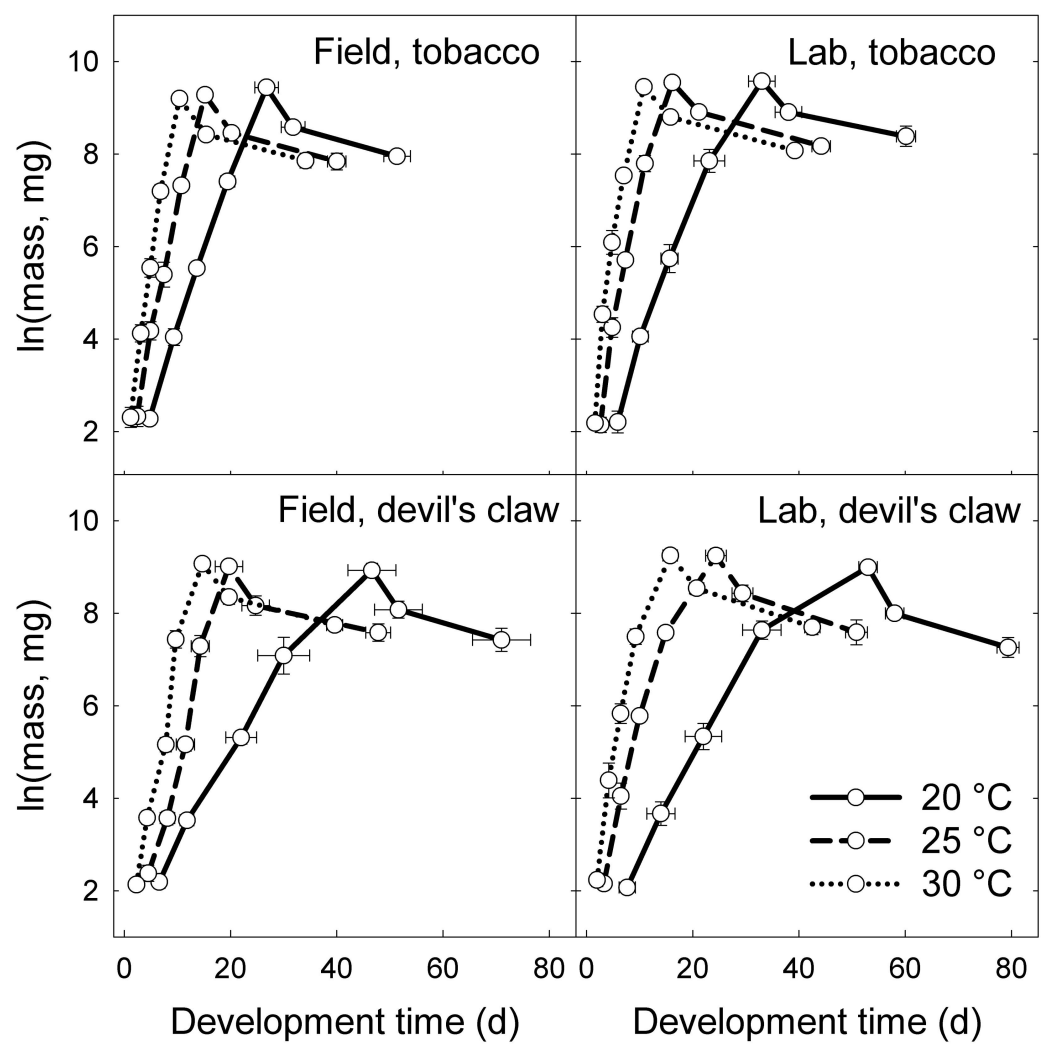

Figure 2: Representations of $\ln$ (mean body mass in $\mathrm{mg}$ ) $\pm 1 \mathrm{SD}$ as a function of mean development time (days) \pm 1 SD at the second, third, fourth, and fifth larval instars and at wandering, pupation, and eclosion for a field population (left) and a laboratory population (right) of female Manduca sexta reared on tobacco (top) and devil's claw (bottom) at $20^{\circ} \mathrm{C}$ (solid lines), $25^{\circ} \mathrm{C}$ (dashed lines), and $30^{\circ} \mathrm{C}$ (dotted lines). Patterns for males were qualitatively similar (fig. A1 in the online edition of the American Naturalist). Individuals with extra (sixth) instars were incorporated into the trajectories for individuals with five instars by omitting the sixth-instar data points.

rate throughout larval growth. These patterns held for individuals within each temperature treatment, although mean trajectories for $M$. sexta reared at cooler temperatures were less steep than those for $M$. sexta reared at warmer temperatures (fig. 2, top; fig. A1, top). In contrast, growth trajectories for $M$. sexta reared on devil's claw were comparatively shallow, with growth slowing considerably during the final larval instar (fig. 2, bottom; fig. A1, bottom). These effects were especially pronounced at cooler temperatures.

Mean development time to pupation was significantly shorter on the high-quality host plant, tobacco, and at warmer rearing temperatures. On each host plant species, mean development times to pupation of the two populations converge at warmer rearing temperatures (figs. 2, 3, A1, A2 in the online edition of the American Naturalist). ANCOVA of development times to pupation detected significant effects of host plant species $\left(F_{1,231}=61, P<\right.$ $.0001)$, temperature $\left(F_{1,231}=350, P<.0001\right)$, the interaction of plant and temperature $\left(F_{1,231}=40, P<.0001\right)$, the interaction of plant and population $\left(F_{1,231}=5.6\right.$, $P=.018)$, and the three-way interaction of plant, population, and temperature $\left(F_{1,231}=5.3, P=.022\right)$ on time to pupation, but no significant effects of population $\left(F_{1,231}=0.095, P=.76\right)$, sex $\left(F_{1,231}=0.74, P=.39\right)$, or the interaction of population and temperature $\left(F_{1,231}=\right.$ $0.23, P=.63)$ were detected. In general, results for development time to wandering and eclosion were qualitatively similar (table A1 in the online edition of the American Naturalist). The presence of significant interaction terms with population indicated that population responses differed across host plant species and temperatures. For M. sexta reared on devil's claw, the slope for development time as a function of temperature is steeper (more negative) for the laboratory population than for the field population. A similar pattern is found for M. sexta reared on tobacco, but the magnitude of the difference between the populations is greater: the laboratory population develops more slowly than the field population at $20^{\circ} \mathrm{C}$ (fig. 3, top; fig. A2, top). 


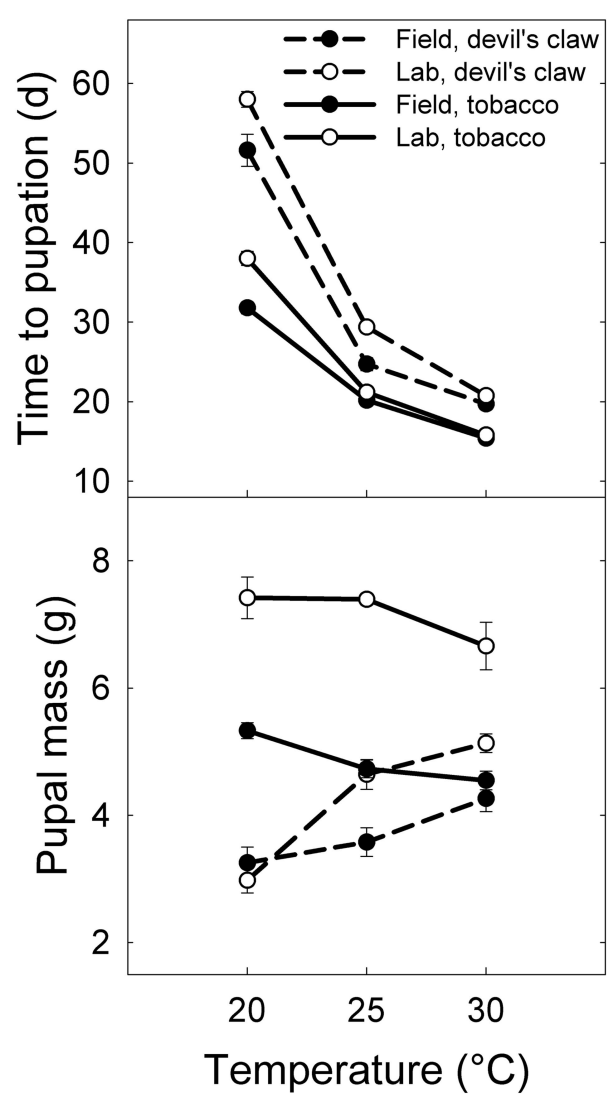

Figure 3: Development time to pupation (days) $\pm 1 \mathrm{SE}$ as a function of temperature $\left({ }^{\circ} \mathrm{C}\right.$; top) and pupal mass $(\mathrm{g}) \pm 1 \mathrm{SE}$ as a function of temperature $\left({ }^{\circ} \mathrm{C}\right.$; bottom $)$ for female Manduca sexta from the field population (filled symbols) and the laboratory population (open symbols) reared on tobacco (solid lines) and devil's claw (dashed lines). Patterns for males were qualitatively similar (fig. A2 in the online edition of the American Naturalist).

Additional developmental responses differed across populations. Field-population and laboratory-population individuals expressed five larval instars (the typical number of instars for M. sexta) on tobacco at all three rearing temperatures, as well as on devil's claw at the warmer temperatures $\left(25^{\circ}\right.$ and $\left.30^{\circ} \mathrm{C}\right)$. In contrast, the induction of an extra (sixth) instar was observed in $30 \%$ of fieldpopulation individuals surviving to eclosion that were reared on devil's claw under the coolest temperature regime $\left(20^{\circ} \mathrm{C}\right)$.

Mean pupal mass was significantly greater for M. sexta raised on the high-quality host plant, tobacco, compared with for those raised on devil's claw. As expected, greater mean pupal mass was achieved at cooler temperatures on tobacco. In contrast, greater mean pupal mass was achieved at warmer temperatures on the low-quality host plant, devil's claw (figs. 2, 3, A1, A2). ANCOVA on pupal mass detected significant effects of host plant species $\left(F_{1,231}=57, \quad P<.0001\right)$, temperature $\left(F_{1,231}=20, \quad P<\right.$ $.0001)$, sex $\left(F_{1,231}=52, P<.0001\right)$, and the interactions of plant and population $\left(F_{1,231}=5, P=.027\right)$, population and temperature $\left(F_{1,231}=4.7, P=.032\right)$, and plant and temperature $\left(F_{1,231}=40, P<.0001\right)$ on body mass at pupation, but no significant effects of population $\left(F_{1,231}=\right.$ $0.98, P=.32$ ) or the three-way interaction between plant, population, and temperature $\left(F_{1,231}=1.5, P=.22\right)$ were detected. In general, results for body mass at wandering and eclosion were qualitatively similar (table A2 in the online edition of the American Naturalist). Here again, significant interactions with population indicated that population responses in body size differed across host plant species and temperature. In M. sexta raised on tobacco, slopes for pupal mass as a function of temperature are similar across populations. In contrast, in those on devil's claw, the thermal reaction norm for body size is steeper (more positive) in the laboratory population relative to the field population (fig. 3, bottom; fig. A2, bottom).

In the models for body size, the interaction term between plant and temperature was significant across all three later developmental stages (wandering, pupation, and eclosion). This indicates that host plant quality alters the slope of thermal reaction norms for final body size (fig. 3, bottom; fig. A2, bottom). Post hoc analyses for each host plant revealed that the slope of pupal mass as a function of temperature was significantly negative when tobacco was the host plant (slope \pm SE: $-85 \pm 27$; $t=$ $-3.2, \mathrm{df}=117, P=.0021)$ but significantly positive on devil's claw (slope \pm SE: $151 \pm 24 ; t=6.4$, df $=119$ $P<.0001)$ when data were pooled across population and sex. Results were qualitatively similar for each combination of plant, population, and sex. In summary, our analyses show that the temperature-size rule (i.e., negative thermal reaction norm for final body size) for $M$. sexta was followed on the high-quality host plant (tobacco), but the rule was reversed on the low-quality host plant (devil's claw), with larger body sizes occurring at warmer rearing temperatures.

\section{Fecundity}

In general, potential fecundity increased with larger body sizes. Females reared on tobacco were larger and more fecund at cooler temperatures; females reared on devil's claw were larger and more fecund at warmer temperatures (fig. 4). ANCOVA revealed significant effects of host plant species $\left(F_{1,91}=10, P=.0018\right)$, population $\left(F_{1,91}=4.5\right.$, $P=.036)$, pupal mass $\left(F_{1,91}=9.9, P=.0022\right)$, the twoway interactions between population and plant $\left(F_{1,91}=\right.$ 11, $P=.0014)$ and between plant and temperature $\left(F_{1,91}=7.7, P=.0068\right)$, and the three-way interaction be- 


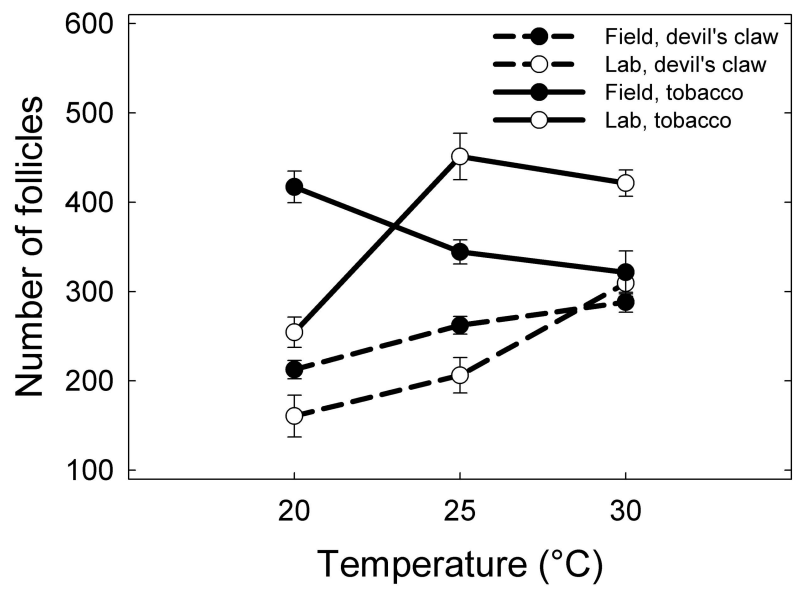

Figure 4: Mean number of follicles in the ovarioles $\pm 1 \mathrm{SE}$ as a function of temperature $\left({ }^{\circ} \mathrm{C}\right.$ ) for females from the field population (filled symbols) and the laboratory population (open symbols) reared on tobacco (solid lines) and devil's claw (dashed lines).

tween plant, population, and temperature $\left(F_{1,91}=12\right.$, $P=.0008)$ on the number of follicles in the ovarioles. No significant effects of temperature $\left(F_{1,91}=1.9, P=.17\right)$ or the two-way interaction between population and temperature $\left(F_{1,91}=3, P=.086\right)$ were detected. In general, results were qualitatively similar when adult mass was used as a covariate in place of pupal mass (table A3 in the online edition of the American Naturalist). The significant threeway interaction between temperature, population, and plant highlights an important exception to this pattern of fecundity generally increasing with larger body size. Laboratory-population females reared at $20^{\circ} \mathrm{C}$ on tobacco had severely reduced potential fecundity despite their large body size under these conditions (fig. 4). The abdomens of these females contained an abnormally high ratio of fat bodies to ovarioles.

\section{Discussion}

The primary goal of this study was to explore the generality of the environmental conditions under which the temperature-size rule is produced. Specifically, we examined the consequences of variation in food quality for the slope of thermal reaction norms using two populations of the tobacco hornworm Manduca sexta. Our results indicate that diet quality can qualitatively affect the slope of thermal reaction norms, leading to resource-dependent reversals of the temperature-size rule.

As expected, $M$. sexta reared on the lower-quality host plant (devil's claw) experienced significant reductions in survival and growth and development rates, longer de- velopment times, and reduced final body size and fecundity (figs. 2, 3, A1, A2). Presumably, reduced fitness on devil's claw results from the nutritional and allelochemical profile of devil's claw being evolutionarily novel for $M$. sexta (S. E. Diamond, S. D. Hawkins, H. F. Nijhout, and J. G. Kingsolver, unpublished manuscript). Similarly, decreasing temperature resulted in decreased growth and development rates and longer development times for M. sexta reared on both host plant species (figs. 2, 3, A1, A2). However, the effects of rearing temperature on survival, final size, and fecundity differed dramatically for the two host plants. Over the temperature range considered here $\left(20^{\circ}-30^{\circ} \mathrm{C}\right)$, survival on tobacco was uniformly high at all rearing temperatures (fig. 1). In contrast, survival on devil's claw decreased with decreasing temperature, suggesting that lower temperatures were stressful for individuals reared on the low-quality host plant but not for those on the high-quality host plant.

The different responses in final body size are of particular interest. On tobacco, M. sexta follow the temperaturesize rule, with smaller final sizes (mass at wandering, pupation, or eclosion) at increasing temperatures. This result was primarily due to increases in body size during the last two larval instars (fig. 2, top; fig. A1, top). Previous results for M. sexta reared on an artificial diet show a similar pattern (Davidowitz et al. 2004; Kingsolver and Nagle 2007). In contrast, M. sexta reared on devil's claw had positive thermal reaction norms for final size, with larger final sizes (mass at wandering, pupation, or eclosion) at warmer temperatures, reversing the temperature-size rule (fig. 3, bottom; fig. A2, bottom). Our results demonstrate that reduced host plant quality can flip the sign of the thermal reaction norm for size, reversing the temperaturesize rule. Importantly, both field and laboratory populations had positive thermal reaction norm slopes when reared on devil's claw. Despite substantial evolved differences in body size between the field and laboratory populations-inadvertent selection for rapid growth contributed to a $50 \%$ increase in body size in the domesticated population (D'Amico et al. 2001) - the laboratory population exhibited qualitatively similar thermal reaction norms when compared with the field population.

One interpretation of our results involves the impact of cold stress on growth and size. Experimental studies that consider a wide range of constant rearing temperatures, primarily with Drosophila, reveal that final size is maximized at an intermediate rearing temperature. Below this temperature, both survival and final size decrease as a result of cold stress; above this temperature, final size decreases with increasing temperature (Moreteau et al. 1997; Karan et al. 1998). In this sense, the temperature-size rule can apply only to nonstressful thermal conditions (van der Have and de Jong 1996; Kingsolver and Huey 2008). The 
lowest temperature treatment in our study $\left(20^{\circ} \mathrm{C}\right)$ is likely not stressful for $M$. sexta being reared on high-quality food resources such as tobacco or an artificial diet, as survival to eclosion in such conditions is quite high $(>80 \%$; fig. 1). In contrast to tobacco, survival to eclosion was quite low in the coldest temperature treatment on devil's claw $(<35 \%)$. This suggests that the reversal of the temperaturesize rule on devil's claw is not a result of general cold stress. Rather, the specific interaction between cooler temperatures and low food quality may represent stressful conditions that lead to the reversal of the temperature-size rule.

Developmental and potential fecundity responses further support this interpretation. Extra (sixth) larval instars were induced only at the cooler temperature treatment on devil's claw. The induction of supernumerary instars is often a response to poor environmental quality (Nijhout 1994; Esperk et al. 2007). Interestingly, extra instars were observed only in the field population that was reared on devil's claw at $20^{\circ} \mathrm{C}$; however, the laboratory population apparently has a reduced developmental capacity for expressing additional instars in response to poor diet quality (S. E. Diamond, S. D. Hawkins, H. F. Nijhout, and J. G. Kingsolver, unpublished manuscript). Similarly, fecundity was reduced for females being reared at the coolest temperatures on devil's claw (fig. 4; see below).

A key assumption in models for the evolution of age and size at reproduction, including models for the evolution of the temperature-size rule, is that body size is directly related to fitness (Stearns and Koella 1986; Atkinson and Sibly 1997); fecundity data provide an important link between body size and fitness. In general, our results for M. sexta support this assumption (see also Davidowitz et al. 2004, 2005). Potential fecundity was greatest under the specific dietary and thermal conditions that contributed to the largest body size: females were most fecund when reared at cooler temperatures on tobacco and warmer temperatures on devil's claw (fig. 4). An important exception to this pattern was found in laboratory-population females reared at $20^{\circ} \mathrm{C}$ on tobacco (fig. 4). Despite having the largest body size of all the treatment groups, the bulk of the body cavity contained only fat bodies (see also Raguso et al. 2007), resulting in severely reduced potential fecundity at cooler temperatures. This reduced fecundity may represent a consequence of laboratory domestication at higher temperatures $\left(25^{\circ}-26^{\circ} \mathrm{C}\right)$, such that $20^{\circ} \mathrm{C}$ may represent a stressful rearing temperature for this population. On the other hand, survival at $20^{\circ} \mathrm{C}$ is quite high for this population when reared on tobacco. It is unlikely that the reduced fecundity we observed reflects a delay in reproduction at lower temperatures, as all M. sexta were maintained at $25^{\circ} \mathrm{C}$ after wandering through metamorphosis when females develop ovarioles and follicles.
Previous work examining the interaction of diet quality and temperature in $M$. sexta has shown that diet quality can alter thermal reaction norms for body size. The effects of temperature on body size have been shown to vary with diet quality when quality was manipulated by the addition of plant allelochemicals to an artificial diet (Stamp 1990, 1994; Yang and Stamp 1996). Similarly, reductions in the protein content of an artificial diet have been shown to alter the slope of thermal reaction norms for final body size in one population of $M$. sexta: relative to that of the population consuming a full-protein diet, the slope of the thermal reaction norm for body size at wandering became less steep (less negative) on a diet with a $39 \%$ reduction in protein content (Petersen et al. 2000). In comparison, it appears that a diet of devil's claw is sufficiently stressful (due to its poor quality for development) to qualitatively alter the slopes of thermal reaction norms and reverse the temperature-size rule. Severe reductions in the nutrient content of artificial diets may also lead to the reversal of the temperature-size rule in laboratory colonies of $M$. sexta (H. F. Nijhout, personal communication).

In $M$. sexta, the reversal of the temperature-size rule may be an important component of this species' adoption of devil's claw as a host plant. We have documented substantial fitness costs associated with $M$. sexta feeding on devil's claw (this article; S. E. Diamond, S. D. Hawkins, H. F. Nijhout, and J. G. Kingsolver, unpublished manuscript). The primary reason for the adoption of devil's claw in spite of these costs appears to be escape from natural enemies (Mira and Bernays 2002; Diamond and Kingsolver 2009). However, if these fitness costs due to poor food quality can be ameliorated by warmer temperatures, as is suggested by our study, then the warmer thermal conditions in the southwestern and southeastern United States, where devil's claw is found, may be an important component of the adoption of this host plant by $M$. sexta.

The frequency with which herbivores encounter lowquality resources in nature may be relatively high: the lack of a relationship between oviposition preference and offspring performance is a widespread pattern in plant-insect interactions (reviewed in Mayhew 1997). The reversal of the temperature-size rule under poor food quality conditions may therefore be a common occurrence. Similarly, novel host plants are often of low quality for herbivores, presenting a barrier to host shifting (Price et al. 1980). This could suggest that host shifts are more likely to occur in warmer climates, where the reversal of the temperaturesize rule would tend to benefit herbivores. Certainly, many additional factors can influence the probability of host shifting: for example, rates of natural enemy attack have been shown to covary with latitude (Hawkins et al. 1997). However, because host shifts and subsequent host race 
formation are important intermediate steps in the process of speciation (Abrahamson and Weis 1997), it is useful to consider how the reversal of the temperature-size rule in low-quality dietary environments may mitigate the costs of switching to novel hosts in warmer climates but exacerbate these costs in cooler climates. Similarly, as thermal environments are being altered by global climate change (Deutsch et al. 2008), the interaction of temperature and food quality could have an appreciable impact on both current and predicted organismal responses to these altered thermal conditions. In general, the extent to which low-quality dietary environments occur in nature and the magnitude of the dietary reductions required to reverse the temperature-size rule are important but unresolved issues.

Empirical and theoretical work examining the role of dietary resources in the context of the temperature-size rule has focused on the effect of temperature on the acquisition, assimilation, and allocation of a given resource (Atkinson 1996; Arendt 1997; Atkinson and Sibly 1997; Robinson and Partridge 2001; Karl and Fischer 2008). In addition, examination of the physiological mechanisms underlying the control of body size and the effects of variation in temperature and diet quality has provided further insight into the basis of the temperature-size rule (Davidowitz and Nijhout 2004; Davidowitz et al. 2004). This work has been important in understanding how the temperature-size rule is produced, but it does not inform us about the range of environmental conditions over which the rule is followed. Here we have demonstrated that diet quality can qualitatively alter the slopes of thermal reaction norms for final body size and reverse the temperature-size rule. As a result, the conditions under which the temperaturesize rule is produced may be less robust than previously thought. Specifically, the temperature-size rule may apply only under conditions of high-quality dietary resources and when other environmental factors are not stressful. Environmental components such as diet quality may therefore be essential to understanding the robustness and limitations to the temperature-size rule.

\section{Acknowledgments}

We thank C. Sorenson for access to the North Carolina State Research Station at Clayton for collecting and useful information on the natural history of Manduca. L. Gilbert and J. Warren provided Manduca eggs from their laboratory colony, as well as advice on rearing Manduca. C. Angell, S. Sheline, and M. Smith helped with the experiments. G. Davidowitz, R. Martin, F. Nijhout, A. Woods, and an anonymous reviewer provided helpful comments on a previous version of the manuscript. The National
Science Foundation provided funding (grants EF-0328594 and IOS-0641179 to J.G.K.).

\section{Literature Cited}

Abrahamson, W. G., and A. E. Weis. 1997. Evolutionary ecology across three trophic levels: goldenrods, gallmakers, and natural enemies. Princeton University Press, Princeton, NJ.

Angilletta, M. J. 2009. Thermal adaptation: a theoretical and empirical synthesis. Oxford University Press, Oxford.

Angilletta, M. J., and A. E. Dunham. 2003. The temperature-size rule in ectotherms: simple evolutionary explanations may not be general. American Naturalist 162:333-342.

Angilletta, M. J., T. D. Steury, and M. W. Sears. 2004. Temperature, growth rate, and body size in ectotherms: fitting pieces of a lifehistory puzzle. Integrative and Comparative Biology 44:498-509.

Arendt, J. D. 1997. Adaptive intrinsic growth rates: an integration across taxa. Quarterly Review of Biology 72:149-177.

Atkinson, D. 1994. Temperature and organism size: biological law for ectotherms? Advances in Ecological Research 3:1-58.

- 1996. Ectotherm life history responses to developmental temperature. Pages 183-204 in I. A. Johnston and A. F. Bennett, eds. Animals and temperature: phenotypic and evolutionary adaptation. Cambridge University Press, Cambridge.

Atkinson, D., and R. M. Sibly. 1997. Why are organisms usually bigger in colder environments? making sense of a life history puzzle. Trends in Ecology \& Evolution 12:235-239.

Awmack, C. S., and S. R. Leather. 2002. Host plant quality and fecundity in herbivorous insects. Annual Review of Entomology 47:817-844.

Berrigan, D., and E. L. Charnov. 1994. Reaction norms for age and size at maturity in response to temperature: a puzzle for life historians. Oikos 70:474-478.

Blanckenhorn, W. U., and M. Demont. 2004. Bergmann and converse Bergmann latitudinal clines in arthropods: two ends of a continuum? Integrative and Comparative Biology 44:413-424.

D'Amico, L. J., G. Davidowitz, and H. F. Nijhout. 2001. The developmental and physiological basis of body size evolution in an insect. Proceedings of the Royal Society B: Biological Sciences 268: 1589-1593.

Davidowitz, G., and H. F. Nijhout. 2004. The physiological basis of reaction norms: the interaction among growth rate, the duration of growth and body size. Integrative and Comparative Biology 44: 443-449.

Davidowitz, G., L. J. D’Amico, and H. F. Nijhout. 2004. The effects of environmental variation on a mechanism that controls insect body size. Evolutionary Ecology Research 6:49-62.

Davidowitz, G., D. A. Roff, and H. F. Nijhout. 2005. A physiological perspective on the response of body size and development time to simultaneous directional selection. Integrative and Comparative Biology 45:525-531.

Deutsch, C. A., J. J. Tewksbury, R. B. Huey, K. S. Sheldon, C. K. Ghalambor, D. C. Haak, and P. R. Martin. 2008. Impacts of climate warming on terrestrial ectotherms across latitude. Proceedings of the National Academy of Sciences of the USA 105:6668-6672.

Diamond, S. E., and J. G. Kingsolver. 2009. Fitness consequences of host plant choice: a field experiment. Oikos. doi:10.1111/j.16000706.2009.017242.x.

Esperk, T., T. Tammaru, and S. Nylin. 2007. Intraspecific variability 
in number of larval instars in insects. Journal of Economic Entomology 100:627-645.

Hawkins, B. A., H. V. Cornell, and M. E. Hochberg. 1997. Predators, parasitoids, and pathogens as mortality agents in phytophagous insect populations. Ecology 78:2145-2152.

Huey, R. B., and P. E. Hertz. 1984. Is a jack-of-all-temperatures a master of none? Evolution 38:441-444.

Karan, D., J. P. Morin, B. Moreteau, and J. R. David. 1998. Body size and developmental temperature in Drosophila melanogaster: analysis of body weight reaction norm. Journal of Thermal Biology 23:301-309.

Karl, I., and K. Fischer. 2008. Why get big in the cold? towards a solution to a life-history puzzle. Oecologia (Berlin) 155:215-225.

Kingsolver, J. G., and R. B. Huey. 2008. Size, temperature, and fitness: three rules. Evolutionary Ecology Research 10:251-268.

Kingsolver, J. G., and A. Nagle. 2007. Evolutionary divergence in thermal sensitivity and diapause of field and laboratory populations of Manduca sexta. Physiological and Biochemical Zoology 80:473-479.

Kingsolver, J. G., J. G. Shlichta, G. J. Ragland, and K. R. Massie. 2006. Thermal reaction norms for caterpillar growth depend on diet. Evolutionary Ecology Research 8:703-715.

Kingsolver, J. G., K. R. Massie, G. J. Ragland, and M. H. Smith. 2007. Rapid population divergence in thermal reaction norms for an invading species: breaking the temperature-size rule. Journal of Evolutionary Biology 20:892-900.

Kingsolver, J. G., G. J. Ragland, and S. E. Diamond. 2009. Evolution in a constant environment: thermal fluctuations and thermal sensitivity of laboratory and field populations of Manduca sexta. Evolution 63:537-541.

Mayhew, P. J. 1997. Adaptive patterns of host-plant selection by phytophagous insects. Oikos 79:417-428.

Mechaber, W. L., and J. G. Hildebrand. 2000. Novel, non-solanaceous hostplant record for Manduca sexta (Lepidoptera: Sphingidae) in the southwestern United States. Annals of the Entomological Society of America 93:447-451.

Mira, A., and E. A. Bernays. 2002. Trade-offs in host use by Manduca sexta: plant characters vs. natural enemies. Oikos 97:387-397.

Moreteau, B., J. P. Morin, P. Gibert, G. Petavy, and J. R. David. 1997. Evolutionary changes of nonlinear reaction norms according to thermal adaptation: a comparison of two Drosophila species. Comptes Rendus de l'Academie des Sciences Series III 320:833841.

Mousseau, T. A. 1997. Ectotherms follow the converse to Bergmann's rule. Evolution 51:630-632.

Nijhout, H. F. 1994. Insect hormones. Princeton University Press, Princeton, NJ.

Nijhout, H. F., G. Davidowitz, and D. A. Roff. 2006. A quantitative analysis of the mechanism that controls body size in Manduca sexta. Journal of Biology 5:1-16.

Partridge, L., and J. A. Coyne. 1997. Bergmann's rule in ectotherms: is it adaptive? Evolution 51:623-635.

Petersen, C., H. A. Woods, and J. G. Kingsolver. 2000. Stage-specific effects of temperature and dietary protein on growth and survival of Manduca sexta caterpillars. Physiological Entomology 25:35-40.

Price, P. W., C. E. Bouton, P. Gross, B. A. McPheron, J. N. Thompson, and A. E. Weis. 1980. Interactions among three trophic levels: influence of plants on interactions between insect herbivores and natural enemies. Annual Review of Ecology and Systematics 11: 45-65.

Radford, A. E., H. E. Ahles, and C. R. Bell. 1968. Manual of the vascular flora of the Carolinas. University of North Carolina Press, Chapel Hill.

Raguso, R. A., T. Ojeda-Avila, S. Desai, M. A. Jurkiewicz, and H. A. Woods. 2007. The influence of larval diet on adult feeding behaviour in the tobacco hornworm moth, Manduca sexta. Journal of Insect Physiology 53:923-932.

Robinson, S. J. W., and L. Partridge. 2001. Temperature and clinal variation in larval growth efficiency in Drosophila melanogaster. Journal of Evolutionary Biology 14:14-21.

Rothschild, W., and K. Jordan. 1903. A revision of the lepidopterous family Sphingidae. Novitates Zoologicae 9(suppl.):1-972.

Small, J. K. 1903. Flora of the southeastern United States. New York. Stamp, N. E. 1990. Growth versus molting time of caterpillars as a function of temperature, nutrient concentration and the phenolic rutin. Oecologia (Berlin) 82:107-113.

. 1994. Interactive effects of rutin and constant versus alternating temperatures on performance of Manduca sexta caterpillars. Entomologia Experimentalis et Applicata 72:125-133.

Stearns, S. C., and J. C. Koella. 1986. The evolution of phenotypic plasticity in life-history traits: predictions of reaction norms for age and size at maturity. Evolution 40:893-913.

van der Have, T. M., and G. de Jong. 1996. Adult size in ectotherms: temperature effects on growth and differentiation. Journal of Theoretical Biology 183:329-340.

Walters, R., and M. Hassall. 2006. The temperature-size rule in ectotherms: may a general explanation exist after all? American Naturalist 167:510-523.

Yamauchi, H., and N. Yoshitake. 1984. Developmental stages of ovarian follicles of the silkworm, Bombyx mori L. Journal of Morphology 179:21-31.

Yang, Y., and N. E. Stamp. 1996. Simultaneous effects of temperature and multiple allelochemicals on the performance of a Solanaceae specialist caterpillar (Manduca sexta). Ecoscience 3:81-92.

Associate Editor: Stephen B. Heard Editor: Donald L. DeAngelis 